\section{Principios orientadores del Modelo Integral de Salud Familiar y Comunitario desde la perspectiva de los usuarios}

\author{
ANGELINA DOIS ${ }^{1, \mathrm{a}}$, AIXA CONTRERAS ${ }^{2, \mathrm{~b}}$, PAULINA BRAVO $^{2, \mathrm{c}}$, \\ ISABEL MORA ${ }^{3}$, GABRIELA SOTO $^{5, \mathrm{~d}}$, CLAUDIA SOLÍS $^{3}$
}

\section{Perception of users about an Integral Model of Family and Community Health care in Santiago, Chile}

\begin{abstract}
Background: The integral Model of Family and Community Health care is based on three essential principles: patient centered care, comprehensive care and continuity of care. Aim: To know the perception of primary care clinic users about the elements that should be considered in a patient centered integrated health care. Material and Methods: Ten males and 31 females aged 18 to 78 years, users of two public family primary care centers participated in focus groups, which were recorded. A qualitative descriptive research design based on content analysis according to Krippendorf was done. Results: Seven issues emerged from the description of patients' experiences: professional-patient relationship, fragmentation of care, continued care with the same professional, promotion and prevention, availability of services and patient records. Conclusions: There are difficulties to install an integral model of family and community health care. The concerns raised by participants should be considered in order to modify the design of these models.
\end{abstract}

(Rev Med Chile 2016; 144: 585-592)

Key words: Patient-Centered Care; Primary Health Care; Public Health.
'Departamento de Salud del Adulto y Senescente, Escuela de Enfermería, Pontificia Universidad Católica de Chile. Santiago, Chile. ${ }^{2}$ Departamento de Salud de la Mujer, Escuela de Enfermería, Pontificia Universidad Católica de Chile. Santiago, Chile. ${ }^{3}$ Departamento de Medicina Familiar, Facultad de Medicina Pontificia Universidad Católica de Chile, Santiago, Chile. ${ }^{4}$ Centro Médico Irarrázaval Red de Salud UC-Christus, Santiago, Chile.

${ }^{a}$ Enfermera-matrona, Magíster en Psicología de la Salud.

${ }^{\mathrm{b}}$ Enfermera-matrona, Magíster en Psicología Social Chile.

'Enfermera. PhD.

'Psicóloga, Magíster en Psicología de la Salud.

Fuente de apoyo financiero: CONICYT: Fondo Nacional de Investigación en Salud (FONIS), Proyecto FONIS SA14ID0025. FONIS no influyó en el diseño del estudio, en la recolección, análisis o interpretación de los datos ni en la preparación, revisión o aprobación del manuscrito.

Recibido el 4 de diciembre de 2015, aceptado el 31 de marzo de 2016.

Correspondencia a: Angelina Dois

Av. Vicuña Mackenna 4860 Macul. Santiago, Chile. adois@uc.cl
E 1 fortalecimiento de la Atención Primaria de la Salud en Chile se basó en un modelo de atención integral y centrado en la promoción y prevención de la salud ${ }^{1,2}$. El Modelo Integral de Salud Familiar y Comunitario se sustenta en tres principios irrenunciables: Centralidad en las personas, Integralidad de la atención y Continuidad del cuidado ${ }^{1,2}$.

La centralidad considera la corresponsabilidad del cuidado de la salud entre el equipo profesional, las personas (sus familias) y la comunidad. Enfatiza los derechos y deberes de los usuarios involucrados y focaliza la atención en la persona y sus necesidades, otorgándoles la información necesaria para participar en las decisiones relativas a su cuidado ${ }^{3}$.

La continuidad se relaciona con la coherencia y conexión que la persona percibe en las distintas acciones relativas al cuidado de su salud, a lo largo de su vida y en distintos escenarios clínicos (desde el domicilio a los centros hospitalarios) ${ }^{2}$.

Finalmente, la integralidad se vincula a la priorización de acciones que garanticen la atención en los tres niveles de complejidad y que articulen acciones de promoción, prevención, curación y rehabilitación desde un abordaje integral de la persona y su familia ${ }^{4}$, lo que exige la disponibilidad de servicios para responder a estas necesidades de salud ${ }^{5}$. 
Estos principios no son atributos de un proveedor sino que se definen a partir de la experiencia de los usuarios 6 . Dado que en Chile este tema ha sido poco explorado, el objetivo de este artículo es describir los elementos que debe considerar la atención en salud integrada, centrada en el usuario y continua desde la percepción de usuarios de Centros de Salud Familiar (CESFAM).

\section{Pacientes y Método}

Este estudio forma parte de un proyecto financiado por el Fondo Nacional de Investigación en Salud de Chile (FONIS SA14ID0025). En el presente artículo se aborda la fase cualitativa inicial del proyecto. La metodología utilizada fue el análisis de contenido de Krippendorff ${ }^{7}$, que permite formular inferencias válidas sobre los datos reunidos, construyendo categorías para describir el fenómeno ${ }^{8,9}$.

\section{Universo y muestra}

Los participantes fueron usuarios de CESFAM de las comunas de La Florida y La Pintana. Los datos se recolectaron entre enero y mayo de 2015, usando muestreo por conveniencia y bola de nieve. Los participantes fueron reclutados por ayudantes de investigación entrenados. Criterios de inclusión: personas mayores de 18 años, atendidas en el mismo CESFAM por al menos 2 años, sin discapacidad o enfermedad grave que impidiera la adecuada comprensión de las preguntas y la manifestación de su opinión o voluntad. Se realizaron cinco grupos focales registrados en grabadora de audio. Se usó un guión de entrevista construido en base a la evidencia disponible y sometido a validación de contenido por expertos (ver Anexo 1).
El criterio de finalización fue la saturación teórica.

Los grupos focales fueron transcritos verbatim. El proceso de análisis consideró:

a) codificación abierta; b) creación de categorías a partir de los códigos obtenidos; c) integración de estas categorías a un esquema teórico más amplio para describir los elementos a considerar en la atención en salud integrada, centrada en el usuario y continua desde la perspectiva de los usuarios. El proceso de análisis consideró el trabajo separado de tres investigadoras y luego la búsqueda de consensos para cada una de las etapas descritas.

El estudio contó con la aprobación del Comité Ético-Científico de la Facultad de Medicina de la Pontificia Universidad Católica de Chile y del Comité de Ética del Servicio Metropolitano Sur-Oriente.

\section{Resultados}

La muestra quedó conformada por 10 hombres y 31 mujeres, de 43,8 años de edad promedio en un rango de 18 a 78 años, usuarios del mismo CESFAM por 10,8 años en promedio.

Del total, 92,7\% asistía de forma regular a sus controles de salud y $29,2 \%$ asistía además regularmente a otras actividades desarrolladas por el Centro (Tabla 1).

El análisis permitió identificar seis dimensiones que debería considerar la atención de salud:

\section{a) Trato al usuario}

Respecto de este punto surgen dos dimensiones de la categoría referidas a lo que los usuarios esperarían recibir de los profesionales de la salud, que se caracterice por (Tabla 2):

Tabla 1. Descripción de los participantes

\begin{tabular}{|lccccccc|}
\hline Sexo & $\begin{array}{c}\mathbf{n} \\
\text { total }\end{array}$ & $\begin{array}{c}\text { Edad } \\
\text { (promedio } \\
\text { de años) }\end{array}$ & $\begin{array}{c}\text { Rango } \\
\text { (años) }\end{array}$ & $\begin{array}{c}\text { Tiempo que ha } \\
\text { sido usuario de } \\
\text { este centro de } \\
\text { salud (promedio } \\
\text { de años) }\end{array}$ & $\begin{array}{c}\text { Asiste en } \\
\text { forma regular } \\
\text { a controles } \\
\text { de salud } \\
\text { (\%) }\end{array}$ & $\begin{array}{c}\text { Asiste en } \\
\text { forma regular } \\
\text { a otras } \\
\text { actividades del } \\
\text { CESFAM (\%) }\end{array}$ & $\begin{array}{c}\text { Asiste frente } \\
\text { a situaciones } \\
\text { de urgencia } \\
\text { (\%) }\end{array}$ \\
\hline Femenino & 31 & 46,7 & $18-72$ & 12,3 & 93,6 & 25,8 & 80,6 \\
\hline Masculino & 10 & 64,7 & $21-78$ & 7,8 & 90 & 30 & 90 \\
\hline Total & 41 & 43,8 & $18-78$ & 10,8 & 92,7 & 29,2 & 90,2 \\
\hline
\end{tabular}




\section{Tabla 2. Trato al usuario}

"Pero en cambio si va una persona y él [médico] va y lo mira a los ojos y dice oh ¿qué, qué le pasó? Y va y le dice "siéntese" le, le hace al menos alguna... Empezando te da la mano cuando entras..."

"Yo creo que hay médicos que usted sale y ni siquiera se dieron cuenta si usted tenía el pelo largo, el pelo corto, si tenía los ojos claros, los ojos oscuros, no se dieron cuenta"

...Yo creo que en el nivel del lenguaje porque igual es importante, o sea los doctores tienen otro tipo de lenguaje... no es, no es el que habla la persona común y corriente... y que venga un doctor y hable así con toda naturaleza por ejemplo con nosotros, yo lo encuentro que... que, que eso es lo principal pienso que... lo que se necesita"

"Un buen doctor que la deje a uno bien, que osea uno, puede que a veces uno va al médico y ni siquiera nos miran nada, ioh! Ojalá el médico nos escuchara, que nosotros pudiéramos decirle todo lo que nos está pasando porque a veces uno queda con tantas cosas de preguntar y llegamos a la casa con lo mismo y no sabemos... nos diera buenos remedios y allí salir uno pucha feliz. Buena atención desde que llegamos y salimos fue bueno"

\section{Tabla 3. Fragmentación de la atención}

"Y le dije: "doctor", le dije: "Sabe que ando con unos dolores en la rodilla", le dije yo "¿Por qué no me da una radiografía?" Le dije yo, "para saber lo que tengo". "No, me dijo, tiene que venir a pedir hora" Y siento que nada le habría costado que me diera una hojita, nada"

"Usted tiene que venir a decirle realmente lo que está sintiendo, si a mí me duele la garganta y me duele la espalda o otra cosa. "iNo!, yo veo una cosa Señora, no con más, así que no venga a decirme que síntomas tiene". Entonces yo encuentro que es malo también"

"Los médicos que aplazan y aplazan. Ellos atienden a una pura persona, una enfermedad específica, la que lleva en ese minuto o sea que si uno tiene cuatro enfermedades tiene que tirar al cara y sello cual le digo al médico"

- Acogida; esperan del profesional una relación que considere patrones básicos de conducta social, como saludar, establecer contacto visual y amabilidad.

- Atención de salud; los usuarios esperan percibir una preocupación genuina por sus problemas y plantean como elementos claves el uso de un lenguaje comprensible y el establecimiento de una relación empática, de confianza, en un tiempo adecuado para exponer su situación de salud/enfermedad y que considere sus opiniones en la solución de sus problemas.

\section{b) Fragmentación de la atención}

Los participantes esperan que el profesional al menos considere los otros problemas que la persona pueda presentar y no sólo uno por cada atención, lo que según los usuarios es la realidad de la consulta de morbilidad actual. Además, relevan la importancia de considerar las necesidades emocionales vinculadas a estos problemas, que muchas veces son más importantes para ellos que la condición que motiva la consulta de salud. Sin embargo, declaran que las experiencias que han vivido, les hacen entender que el sistema de salud funciona centrado en los problemas y no en las personas (Tabla 3 ).

\section{c) Continuidad de acceso a los profesionales}

Los usuarios describen la importancia que tiene la posibilidad de atenderse siempre con los mismos profesionales, que permite construir una relación de confianza con el médico y el resto del equipo. Por ello, defienden su derecho a elegir al profesional que los atienda. Además, esto permite que los profesionales estén al tanto de la situación de salud de la persona, pudiendo hacerle seguimiento al plan terapéutico (Tabla 4).

\section{d) Promoción y prevención}

Los participantes describen la necesidad de contar con actividades promocionales y preventivas en los centros de salud, que complementen la atención de salud que reciben por distintos problemas (Tabla 5). 


\section{Tabla 4. Continuidad de acceso a los profesionales}

"Eso está mal porque yo encuentro que si una matrona le está viendo el problema, después la mandan a otro, entonces hay que empezar todo de nuevo... contar toda la historia... Estarle explicando todo el caso de nuevo... Entonces uno se cansa y sabe que le dije yo a la niña, le dije: "a mí me ve la doctora -no, me dijo, porque esto no es particular dijo, aquí le tiene que tocar con el médico que hay no más"

“... Pero me sentía cómoda y me decía lo que me estaba pasando y todo poh, o lo que iba pasando en el momento que iba creciendo mi guata. Y ahora ya no se puede elegir al matrón que uno quiera elegir que la atienda"

"La última vez que estuve que me tocaba con otra profesional y... pero no sé por qué me la cambiaron"

\section{Tabla 5. Promoción y prevención}

"Lo importante sería de que hubiese y no sé habrá aquí una dietista que enseñe a los pacientes, acá a los que habitualmente venimos, a alimentarse mejor, porque usted sabe y por lo menos yo sé que la alimentación es la base de las enfermedades la mala alimentación"

"Entonces, por lo menos a mí, pienso que dentro de la salud integral está esos talleres en que si la gente los sabe aprovechar, sí son provechosos"

"Y esos talleres son para... (usuario) para prevenir y para las personas que están ya con diabetes o sea para cuidarse"

\section{e) Disponibilidad de servicios}

Según los participantes, un elemento central para asegurar la calidad de la atención es el acceso oportuno a prestaciones que se otorgan en otros niveles de complejidad, particularmente interconsultas, exámenes y procedimientos terapéuticos, incluyendo los quirúrgicos. Además, describen tiempos de espera prolongados y/o escases de prestadores lo que muchas veces los obliga a atenderse en otras comunas utilizando direcciones falsas o a resolver sus problemas en la red de prestadores privados, situación fuera del alcance de muchos, básicamente por los costos económicos asociados.

Adicionalmente, la disponibilidad y acceso a diferentes prestaciones dentro del CESFAM es considerada por ellos como un componente central de la atención en salud y plantean que las dificultades en este punto se dan en parte por la escases de profesionales para satisfacer las demandas de la población, lo que provoca un menor número de consultas y de tiempo asignado a cada una de ellas. Además, proponen un agendamiento diferenciado según la edad del consultante, asegurando la atención de personas mayores, niños y población general sin necesidad de largas esperas para conseguir un número de atención (Tabla 6).

\section{f) Registros de pacientes}

Los participantes son enfáticos en afirmar que el médico debe contar con toda la información disponible respecto del usuario para que pueda otorgarles una atención de calidad y a la vez hacer seguimiento de otras atenciones de las que hayan sido objeto en el mismo Centro o dentro de la red de salud. Plantean la necesidad de contar con una ficha clínica en línea, que reúna todos los antecedentes del usuario, que permitirá también abordar la dificultad que se le genera al usuario para transmitir resultados de interconsultas $y$ atenciones de salud entre un profesional y otro ya que en su mayoría no entienden y/o no retienen la información oral recibida de los profesionales, sumado al hecho que esta información no siempre se les entrega por escrito (Tabla 7).

\section{Discusión}

El sistema de salud chileno está basado en la Atención Primaria, de ahí la relevancia de acciones fortalecedoras de sus principios. El primero, es la atención centrada en el usuario (ACU), que considera como elemento fundamental según los participantes, el trato recibido por los profesionales, lo que sido reportado en otros estudios, 


\section{Tabla 6. Disponibilidad de servicios}

Disponibilidad de servicios dentro de la red de salud

Disponibilidad de servicios dentro del centro de atención primaria
"Pero ahora no hay nada, hay que hacerse las radiografías particulares, hay que hacerse todo particular. O sea no hay beneficios para uno yo encuentro... lo otro también que cuando, por ejemplo... a mí me dieron una interconsulta también para las rodillas y usted tiene que esperar que la llamen. Resulta que llevaré siete años y todavía no me llaman, sí es verdad"

"Ahora me mandó al kinesiólogo, ahora tengo que ir allá al San Rafael porque acá no hay kinesiólogo"

A mí se me cayó un diente por acá y por una movía que hizo mi hermana yo me los pude colocar allá en Puente Alto porque acá nunca tenía hora, nunca alcancé hora, nunca

“En primer lugar mucha la espera porque hay uno o dos médicos y a veces llegan como veinte o treinta personas, entonces no da abasto"

“La asistente social a las 10 de la mañana tiene que entregar a la psicóloga, tiene que pasarle su oficina... la podóloga tiene que atender después de las 7 de la tarde porque no tiene oficina donde atender. No hay, no hay, no hay box para médicos acá en este consultorio"

"Acá vienen 150 personas, dan 30 números en la mañana, porque hay un puro médico"

"Estarse levantando a las cinco de la mañana o antes para pedir un número para médico y todas esas cosas, antes daban más horas, ahora a veces dan treinta números para tanta gente que hay esperando

Tabla 7. Registro de la información

"Ahí debe ser en forma interna... eeehh se le realizaron la radiografía todo bien en caso de urgencia vuelva el... entonces así el médico también cuando abra el computador y lea en la ficha de ella va a saber que ya fue y en qué condiciones ella ya está"

"(usuario 1) Debiera haber un seguimiento pa' cualquier caso, qué diagnóstico de los médicos si resultó o no resultó la cosa... lo vamos a conversar esto con la doctora $x$, porque... (usuario 2) debiera haber una ficha, así un antecedente un eeeh de toda la red de salud que uno consulta de... de servicio público, debiera haber una, una ficha"

"En cambio si hubiera una ficha clínica, no habría ese problema porque habría... la abriría un médico y sabría a quién está, está tratando... qué son, cuáles son los seguimientos que se le han hecho y todo, todo un tema, mayor control y todo eso, entonces..."

destacándose la manera en que los profesionales se aproximan a los usuarios, les saludan, personalizan sus interacciones y demuestran un trato respetuoso e interesado ${ }^{3,9,10}$, pero según los participantes del estudio esta característica sigue en deuda, relevando la necesidad de contar con un trato acogedor, personalizado y empático que transforme el encuentro con el profesional, en un espacio de apoyo y co-construcción para la solución a los problemas de salud que presentan.

El trato respetuoso considera también el reconocimiento de valores y creencias del usuario y su participación activa en las decisiones vinculadas a su salud. Esto se relaciona directamente con marco conceptual de la APS que desde la perspectiva biopsicosocial, reconoce al usuario como persona y enfatiza en el poder y responsabilidad compartidos en el cuidado de la salud, construido sobre una alianza terapéutica profesional-usuario ${ }^{11}$ que promueve el empoderamiento y la participación de las personas en la toma de decisiones que conciernen a su salud ${ }^{3,12-14)}$. Estos elementos se vinculan con mejoras en los índices de satisfacción usuaria, en los resultados clínicos a mediano y largo plazo y en la organización y coordinación de los servicios que ofrecen los prestadores de salud, lo que se traduce en una mejor calidad de atención y disminución de los costos globales de atención, Además, no debe olvidarse que el marco legal vigente destaca el trato digno al usuario como 
uno de los derechos fundamentales de las personas en relación a la atención de salud, situándolo por sobretodo como una obligación del profesional ${ }^{15}$.

Otro componente de la ACU es la no fragmentación de la atención, que significa considerar globalmente la situación del usuario, más allá de la patología. La literatura destaca que esta aproximación tiene un potencial organizador de la atención en salud y permite al usuario experimentar una atención en salud transparente, individualizada ${ }^{16} y$ congruente con los servicios otorgados en función de sus necesidades ${ }^{17}$, lo que claramente se opone a la experiencia compartida por los participantes del estudio y respecto de la cual manifiestan su disconformidad.

El segundo principio orientador de la APS es la integralidad, entendida como el suministro continuo, articulado y de calidad de un amplio rango de prestaciones para las personas y sus familias a lo largo del ciclo vital2 ${ }^{2,418}$, y que exige la complementariedad de los servicios entre establecimientos de salud, la red de instituciones de salud y los procesos de coordinación interinstitucionales, ya que debe ser desarrollada en co-responsabilidad entre el sector salud y otros sectores sociales ${ }^{4,18}$.

La atención integral busca mantener a las personas sanas, por ello todo contacto debe considerar acciones promocionales y preventivas, rompiéndose así con el esquema tradicional de abordaje patocéntrico de la salud ${ }^{4}$ y que los participantes del estudio perciben como una la necesidad. Esto se orienta hacia los lineamientos propuestos por la política nacional vigente como también por diferentes estudios internaciona$\operatorname{les}^{2,19-21}$, que han demostrado la vinculación entre los resultados clínicos en distintas poblaciones con la incorporación de acciones de promoción y autocuidado durante la atención de salud ${ }^{3}$.

La OMS ha reiterado lo ventajoso de los programas integrales, ya que hace más eficiente el uso de recursos, disminuye la relación costo-efectividad, optimiza el uso del tiempo del equipo de salud y se convierte en una fuerza unificadora en la red intersectorial relacionada, como educación y asistencia social entre otras. Asimismo, evita la duplicidad de actividades generadas en programas verticales pudiendo integrarse la información sanitaria, en favor de la eficiencia de la gestión del sistema ${ }^{22}$.

Sin embargo, la experiencia de los participantes da cuenta de un sistema que presenta dificultades para el acceso a la atención de salud como también para proporcionar los servicios requeridos para su cuidado, tanto en los centros de salud primarios como en otros niveles de atención y que según ellos, ha caracterizado históricamente la atención de salud, dando cuenta de una clara dificultad para la implementación de este principio en el modelo de salud actual.

Por último, el tercer principio orientador del modelo es la continuidad de la atención, entendida como la coherencia que experimentan los usuarios respecto de la atención de salud que reciben a lo largo de su vida en distintos escenarios de la red de salud ${ }^{2,23,24}$. Uno de sus componentes destacado por los participantes del estudio, se refiere a la continuidad relacional, es decir, la posibilidad de acceder a la atención de salud con un mismo profesional (o equipo de salud), lo que permite construir relaciones de confianza y dar continuidad real al plan terapéutico. Este tipo de vínculo es altamente valorado en el contexto de la APS ya que provoca la fidelización del usuario con su proveedor de salud, y estimula la responsabilidad del proveedor por su paciente ${ }^{6,13,25}$.

El segundo componente de este principio es la continuidad informacional entre los servicios de atención, que permita establecer mecanismos de transferencia de la información desde y hacia otras fuentes involucradas en la atención de las personas ${ }^{6,13,25,26}$, con instrumentos de registro que permitan el seguimiento del plan de atención de la persona y la implementación de planes de identificación y gestión de necesidades de la población ${ }^{27}$.

$\mathrm{Al}$ año 2013, cerca de 60\% de la APS en Chile contaba con algún tipo de registro clínico electrónico $(\mathrm{RCE})^{28}$. Pese a este notable avance, aún existe una importante brecha en los sistemas de información que dificulta la comunicación entre los distintos niveles de atención en el país. Los participantes de este estudio dan cuenta de esta situación manifestando abiertamente la necesidad de contar con sistemas en línea que reúnan todos sus antecedentes, para una mejor atención y seguimiento en el CESFAM donde son atendidos habitualmente, como en otros puntos de la red de salud, ya que no se encuentran habilitados para transmitir a los profesionales de la salud, los antecedentes técnicos de las atenciones y tratamientos prescritos.

En conclusión, el Modelo de Salud Integral Familiar y Comunitario se sostiene en tres princi- 
pios irrenunciables que dan cuenta de la filosofía de la APS, relevando la participación del usuario como agente activo en la mantención de su salud y en el proceso terapéutico de su enfermedad. Los principios orientadores del modelo de salud se de- finen a partir de la experiencia de los beneficiarios y no de los proveedores de salud, es por esto que los resultados de este estudio, pueden servir para orientar a los equipos de salud en el fortalecimiento del Modelo en las distintas realidades locales.

\section{Anexo 1. Guión Grupo Focal}

a. Según su última atención de salud ¿Qué es para usted lo más relevante o importante en su atención de salud?

b. ¿Cuáles diría usted que fueron los momentos claves de su atención? Desde que ingresa al centro de salud hasta que sale

c. En términos generales ¿Qué es lo más importante para usted cuando acude a una consulta de salud en este centro de salud?

d. ¿Qué aspectos definen para usted una "buena atención de salud"?

e. Si pudieran describir los elementos centrales de su atención. ¿Cuáles serían aquellos aspectos que Ud. destacaría (sea por bueno o por malo de acuerdo a su apreciación)?

f. Respecto de la atención de salud centrada en la persona ¿Qué es lo más importante para usted? A su juicio ¿qué ventajas y desventajas tendría que un mismo profesional lo atendiera cada vez que necesita una atención de salud?

g. Respecto de la atención de salud integral ¿Qué es lo más importante para usted?

h. Respecto de la continuidad de la atención de salud ¿Qué es lo más importante para usted?

\section{Referencias}

1. Organización Panamericana de la Salud. Hacia un modelo de atención integral a la salud basado en la Atención Primaria de Salud. Washington DC: Organización Panamericana de la Salud, 2012.

2. Ministerio de Salud. Orientaciones para la implementación del Modelo de Atención Integral de Salud familiar y comunitaria. Chile: Ministerio de Salud, 2013.

3. Robinson J, Callister L, Berry J, Dearing KA. Patient-centered care and adherence: Definitions and applications to improve outcomes. J Am Assoc Nurse Pract 2008; 20: 600-7.

4. Aguilera C. O desafio da integralidade segundo as perspectivas da vigilância da saúde e da saúde da família. Ciênc Saúde coletiva 2003; 8 (2): 569-84.

5. Organización Panamericana de la Salud. Redes integradas de servicios de salud. Concepto, opciones de política y hoja de ruta para su implementación en las Américas. Washington, D.C: Organización Panamericana de la Salud, 2010.

6. Haggerty J, Reid R, Freeman G, Starfield B, Adair C, McKendry R. Continuity of care: a multidisciplinary review. BMJ 2003; 32: 1219-21.
7. Krippendorff K. (2004). In Krippendorff K. (Ed.), Content analysis, an introduction to its methodology (second ed.). Thousand Oaks, California: Sage Publications.

8. Elo S, Kyngäs H. The qualitative content analysis. JAN 2007; 62 (1): 107-15.

9. Beach M, Saha S, Cooper L. The role and relationship of cultural competence and patient-centeredness in health care quality. Commonwealth Fund 2006; (960): 1-22.

10. Morgan S, Yoder L. A concept analysis of person-centered care. J Holist Nurs 2012; 30 (1): 6-15.

11. Mead N, Bower P. Patient-centeredness: A conceptual framework and review of the empirical literature. Soc Sci Med 2000; 51: 1087-110.

12. Kitson A, Marshall A, Bassett K, Zeitz K. What are the core elements of patient-centred care? A narrative review and synthesis of the literature from health policy, medicine and nursing. JAN 2013; 69 (1): 4-15.

13. Institute of Medicine. Crossing the Quality Chasm: A New Health Systemfor the 21st Century. Washington, DC: National Academy Press, 2001.

14. Hobbs J. A dimensional analysis of patient-centered care. Nurs Res 2009; 58 (1): 52-62.

15. Ley $\mathrm{N}^{\circ} 20.584$ Regula los derechos y deberes que tienen 
las personas en relación con acciones vinculadas a su atención en salud, 2012.

16. Rathert C, Wyrwich M, Boren S. Patient-Centered Care and Outcomes: A Systematic Review of the literature. Med Care Res Rev 2013; 70: 351-79.

17. Montenegro H, Suárez J. Redes Integradas de Servicios de Salud: conceptos, opciones de política y hoja de ruta para su implementación en las Américas. Wasington, D.C.: Organización Panameriacana de la Salud, 2010.

18. Acosta N, Vega R. El caso de la implementación de la estrategia de Atención Primaria Integral en Salud (APIS). Rev Gerenc Polit Salud 2008; 7 (14): 125-44.

19. Ministerio de Salud de Guatemala. Red de Servicios de Salud del MSPAS. Guatemala: Ministerio de Salud, 2010.

20. Rosas A, Zárate V, Cuba M. Atributos de la Atención Primaria de Salud (A.P.S): Una visión desde la Medicina Familiar. Acta med peruana 2013; 30 (1). Disponible en http://www.scielo.org.pe/scielo.php?script=sci_arttext\&pid=S1728-59172013000100008 [Consultado el 6 de marzo de 2015].

21. Villegas O, Castillo A, Montero D, Holthuis K, Álvarez A, Freer J, et al. Bases para la Atención Médica Integral. San José, Costa Rica: Editorial Nacional de Salud y Seguridad Social (EDNASSS), 2003.

22. Organización Mundial de la Salud. La Atención Prima- ria en Salud, más necesaria que nunca. Informe sobre la salud en el mundo 2008. Ginebra: Organización Mundial de la Salud, 2008.

23. Sweeney A, Rose D, Clement S, Jichi F, Jones I, Burns T, et al. Understanding service user-defined continuity of care and its relationship to health and social measures: a cross-sectional study. BMC Health Serv Res 2012; 12 (145). Disponible en http://www.biomedcentral.com/ content/pdf/1472-6963-12-145.pdf [Consultado el 18 de mayo de 2015].

24. Uijen A, Schers H, Schellevis F, van den Bosch W. How unique is continuity of care? A review of continuity and related concepts. Fam Pract 2012; 29: 264-71.

25. Reid R, Haggerty J, McKendry R. Defusing the confusion: concepts and measures of continuity of healthcare. Ottawa: Canadian Health Services Research Foundation, 2002. Disponible en: http://www.cfhi-fcass.ca/Migrated/ $\mathrm{PDF} /$ ResearchReports/CommissionedResearch/cr_contcare_e.pdf [Consultado el 11 de mayo de 2015].

26. Starfield B. Is primary care essential? Lancet 1994; 344 : 1129-33.

27. Ministerio de Salud (2013). Informe público de avance SIDRA. Chile: Ministerio de Salud. Disponible en http://www.salud-e.cl/wp-content/uploads/2013/11/ Informe-Pu\%CC\%81blico-SIDRA-2013-03.pdf [Consultado el 8 de septiembre de 2015]. 